\title{
Eculizumab in atypical hemolytic uremic syndrome: long-term clinical course and histological findings
}

\author{
Sibylle Tschumi • Mathias Gugger • Barbara S. Bucher • \\ Magdalena Riedl • Giacomo D. Simonetti
}

Received: 10 July 2011 /Revised: 28 July 2011 / Accepted: 28 July 2011 /Published online: 30 August 2011

(C) IPNA 2011

\begin{abstract}
Atypical hemolytic uremic syndrome (aHUS) is a thrombotic microangiopathy associated with defective regulation of the alternative complement pathway. The prognosis for patients with aHUS is poor, and plasma exchange represents the first-line therapy. Eculizumab is a humanized monoclonal anti-C5 antibody that prevents the activation of the terminal complement pathway. Here, we report the case of a 9-year-old girl with frequent relapsing aHUS due to heterozygous factor $\mathrm{H}$ mutation who was initially treated with plasma exchange three times per week with $150 \%$ plasma exchange volume. This treatment frequently caused allergic reactions and school absences. Because any reduction in the frequency of plasma exchange immediately induced relapses of the aHUS, treatment with eculizumab, $600 \mathrm{mg}$ every 2 weeks, was started and plasma exchange completely stopped. On this drug regimen the patient showed no evidence of disease activity during a period of more than 24 months. Renal function improved, proteinuria disappeared, the number of antihypertensive medications could be decreased, and the quality of life increased substantially. The inhibition of the terminal complement pathway by eculizumab was also confirmed by renal biopsy, which showed the absence of thrombotic
\end{abstract}

S. Tschumi • B. S. Bucher • G. D. Simonetti $(\bowtie)$

Division of Paediatric Nephrology, Children's Hospital,

University of Bern,

3010 Berne, Switzerland

e-mail: giacomo.simonetti@insel.ch

\section{Gugger}

Department of Pathology, University of Bern,

Bern, Switzerland

M. Riedl

Department of Pediatrics, Medical University Innsbruck, Innsbruck, Austria microangiopathy 2 months after the initiation of eculizumab therapy. This case illustrates the long-term favorable outcome of aHUS with eculizumab treatment.

Keywords Atypical hemolytic uremic syndrome. Factor H · Complement · Eculizumab · Children

\section{Introduction}

Atypical hemolytic uremic syndrome (aHUS) is a thrombotic microangiopathy characterized by hemolytic anemia, thrombocytopenia, and renal failure [1] and has been associated with defective regulation of the alternative complement pathway [2, 3]. The prognosis for patients with aHUS associated with factor $\mathrm{H}$ mutation is poor, with frequent relapses; the majority of cases progress to end stage renal disease requiring dialysis and renal transplantation [4]. Plasma exchange is the first-line treatment to rescue aHUS episodes and prevent relapses [5], but the long-term acceptance and efficacy of this treatment is controversial [5]. Eculizumab (Soliris; Alexion Pharmaceuticals, Cheshire, $\mathrm{CT}$ ) is a humanized monoclonal anti-C5 antibody that prevents the activation of the terminal complement pathway and hinders the generation of proinflammatory $\mathrm{C} 5 \mathrm{a}$ and the potentially lytic C5b-9 (membrane attack) complex. It has already been approved for the treatment of paroxysmal nocturnal hemoglobinuria, another complement disorder [6]. Recent case reports have demonstrated the efficacy of eculizumab in both transplanted and non-transplanted patients with aHUS [7-13].

Here, we describe the long-term clinical and histological features of a child with aHUS who has been successfully treated with eculizumab over a long observation period of 24 months. 


\section{Case report}

A 9-year-old girl with frequent relapsing aHUS had to be treated with plasma exchange three times per week with $150 \%$ plasma exchange volume in order to limit aHUS exacerbations. Genetic analysis disclosed a heterozygous factor H mutation in SCR 10 (Cys611Tyr). This mutation has not yet been described in the literature, and to date only one mutation in SCR 10, with unclear significance, has been described in the "Factor H Mutation Database" (http:// www.fh-hus.org). Attempts to give plasma infusions or to reduce the frequency of plasma exchange from three times per week to two times per week resulted in relapses at days 22 and 44, respectively. During these relapses, moderate thrombocytopenia, hemolytic anemia with modest increases in lactate dehydrogenase (LDH), a rise in the serum creatinine level, and worsening hypertension were observed (Fig. 1). Given the normal concentration of factor $\mathrm{H}$ (427 mg/l, norm 345-590 mg/l) and the patient's nonresponse to plasma infusions, we assume that this mutation induces a dysfunctional protein. However, the treatment regimen of plasma exchange therapy three times per week up to day 126 caused frequent allergic reactions and frequent school absences, leading us to initiate treatment with eculizumab, $600 \mathrm{mg}$ every 2 weeks. With this drug regimen and without plasma exchange there was no evidence of disease activity for over 24 months, despite the occurrence of some minor viral infections. The renal function improved [estimated glomerular filtration rate (eGFR) rose from 40 to $56 \mathrm{ml} / \mathrm{min} / 1.73 \mathrm{~m}^{2}$ ], proteinuria completely disappeared, the number of antihypertensive drugs decreased from five to three with a substantial improvement of myocardial hypertrophy and regression of hypertensive retinopathy, the erythropoietin amount could be reduced, and the quality of life increased substantially (Fig. 1). The child was vaccinated against Neisseria meningitidis (meningococcal polysaccharide vaccine against $N$. meningitidis type A,C,W,Y; Mencevax ACWY, GlaxoSmithKline, Brentford, UK) prior to the start of eculizumab treatment. The treatment was well tolerated without relevant side effects. At the beginning of the treatment, the patient complained for 2 weeks about rhinitis, which completely disappeared over time. During the therapy with eculizumab, the whole complement function (CH50) was reduced to $25-30 \%$ of its pretreatment value. It was, therefore, not completely blocked, but its ability to activate the terminal complement complex (TCC) in serum was considerably suppressed, as has been described elsewhere [14] (Fig. 1). A renal biopsy performed during the first relapse of aHUS revealed the presence of thrombotic microangiopathy; in contrast, thrombotic microangiopathy was absent in the renal biopsy performed 2 months after the start of eculizumab therapy although glomerular scarring and fibrosis were present, likely representing chronic lesions of the previous aHUS relapses. In addition, glomerular C5b-9 deposits were absent during the eculizumab treatment based on immunofluorescence testing.

\section{Discussion}

Patients with aHUS due to factor $\mathrm{H}$ mutations have a poor prognosis with fast progression to end stage renal disease [4]. Plasma exchange is recommended for the acute management of aHUS [5], but little is known about the long-term treatment options. In order to stabilize disease activity, our patient required frequent (3 times per week) plasma exchange, and relapses were observed when the frequency was reduced or plasma infusions performed. This invasive procedure, which required a dialysis central venous catheter, caused frequent allergic reactions and school absences. We therefore decided to initiate therapy with eculizumab, a terminal complement inhibitor, which was able to avoid the recurrence of aHUS without plasma exchange and provided a long-term control of complement activity. During the therapeutic regimen with eculizumab, the renal function partially improved, and the amount of antihypertensive drugs and erythropoietin amount could be decreased. Renal function did not completely normalize, probably because the previous relapses of aHUS induced scarring and fibrosis of the glomeruli, as demonstrated by the biopsy findings. During the period of eculizumab therapy, the whole complement activity (CH50) was between $25-30 \%$ of the pretreatment value and therefore not completely blocked. However, we also measured the ability of eculizumab to activate the TCC [14], which was considerably suppressed, possibly indicating an appropriate dosage of eculizumab. Moreover, despite the non-blockade of the complement, as demonstrated by continuing $\mathrm{CH} 50$ activity, the absence of relapses even with common viral infections during the follow-up with eculizumab also demonstrated an adequate complement inhibition by this drug. It therefore remains unclear which biological criteria should be used to show adequate control of complement activation. In this case, the ability to activate TCC would seem to be the best reflection of the clinical follow-up.

Since the first descriptions of the effect of eculizumab on aHUS published in 2009 [8, 9, 11], nearly 20 reports have documented the positive effect of eculizumab in aHUS [713]. Some cases also showed an improvement of renal function after therapy with eculizumab [8, 12], but others were unable to reverse the chronic renal failure, probably because of irreversible glomerular scarring [9]. To date, no side effects have occurred in our patient or in the cases described in the literature. Prudence is recommended, 

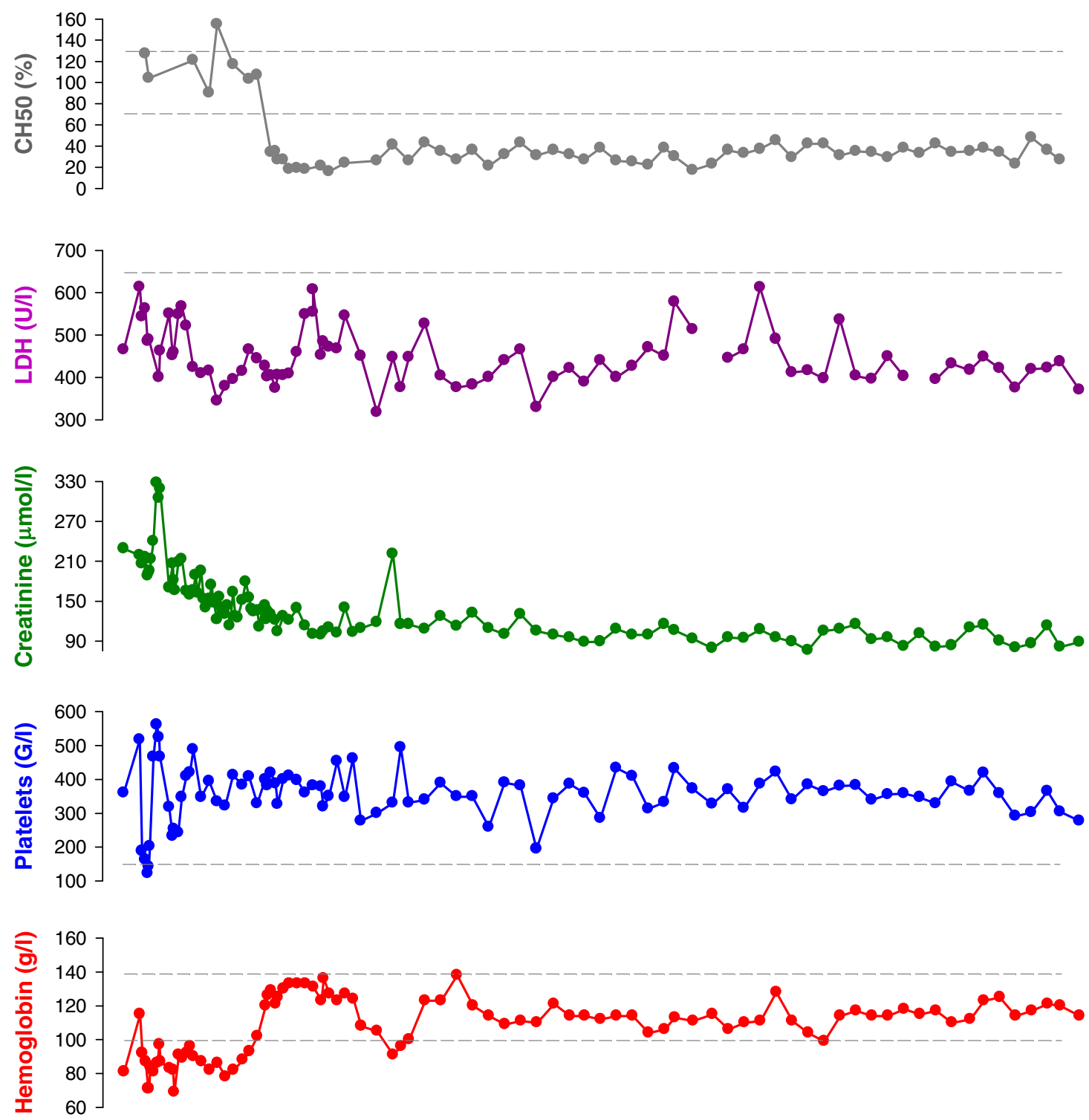

Eculizumab (600 mg every 2 weeks)

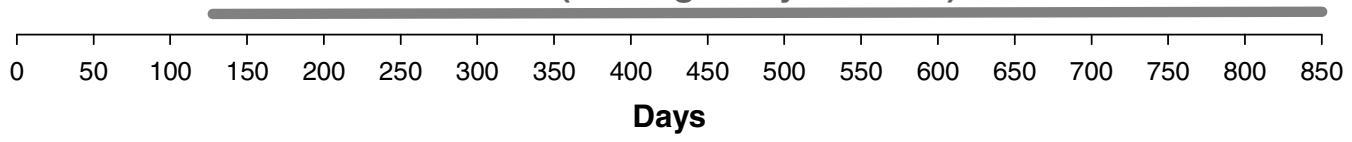

Fig. 1 A 9-year-old girl with relapsing atypical hemolytic uremic syndrome (aHUS) due to a factor $\mathrm{H}$ mutation was treated with plasma exchange three times per week with $150 \%$ plasma exchange volume. A reduction in the plasma exchange frequency induced relapses of aHUS, manifesting as increased blood pressure, worsening of the renal function, thrombocytopenia, anemia, and increased lactate dehydrogenase levels $(L D H)$. After this period, the patient received eculizumab. Thereafter, no plasma exchange was performed. Hemoglobin

however, because this treatment increases the risk for infection by encapsulated organisms, such as $N$. meningitidis. Meningococcal vaccination is necessary at least 2 weeks prior to the initiation of therapy with eculizumab. However, the most frequent meningococcal strain in Europe is serotype $\mathrm{B}$, which is not covered by the vaccines that are levels remained stable (with less erythropoietin) without signs of hemolysis (LDH) and with normal platelet counts; the renal function progressively improved (creatinine). The fall in hemoglobin on day 240 is due to the quick reduction of erythropoietin and not a sign of relapse of aHUS. After the commencement of eculizumab, the whole complement function $(\mathrm{CH} 50)$ was substantially suppressed at about $25-30 \%$ of the pretreatment level. The dashed lines represent the lower or upper limit of the normal ranges

currently available; therefore, it seems opportune to recommend prophylactic penicillin $\mathrm{G}$ therapy, as stressed in a recently published letter [15].

The inhibition of terminal complement activity by eculizumab was also confirmed in our patient by renal biopsy, which showed the absence of thrombotic micro- 
angiopathy and glomerular C5b-9 deposits, based on immunofluorescence testing, 2 months after the initiation of eculizumab therapy. However, the absence of these deposits should be taken cautiously due to the poor sensitivity and specificity of the available antibodies.

In conclusion, this case illustrates the long-term (24 months) favorable outcome of aHUS following treatment with eculizumab, indicating, in agreement with other cases reported in the literature, that eculizumab is a valuable alternative for the long-term treatment of patients with relapsing aHUS. However, it remains unclear whether it will be possible to reduce the eculizumab doses or completely stop the treatment during follow-up. Clinical trials are ongoing (clinicalTrials.gov identifier NCT 01193348) to assess the long-term efficacy and safety of eculizumab in children with aHUS.

Potential conflict of interest None.

\section{References}

1. Gasser C, Gautier E, Steck A, Siebenmann RE, Oechslin R (1955) hemolytic-uremic syndrome: Bilateral necrosis of the renal cortex in acute acquired hemolytic anemia. Schweiz Med Wochenschr 85:905-909

2. Kavanagh D, Goodship T (2010) Genetics and complement in atypical hus. Pediatr Nephrol 25:2431-2442

3. Loirat C, Noris M, Fremeaux-Bacchi V (2008) Complement and the atypical hemolytic uremic syndrome in children. Pediatr Nephrol 23:1957-1972

4. Sellier-Leclerc AL, Fremeaux-Bacchi V, Dragon-Durey MA, Macher MA, Niaudet P, Guest G, Boudailliez B, Bouissou F, Deschenes G, Gie S, Tsimaratos M, Fischbach M, Morin D, Nivet H, Alberti C, Loirat C (2007) Differential impact of complement mutations on clinical characteristics in atypical hemolytic uremic syndrome. J Am Soc Nephrol 18:2392-2400
5. Ariceta G, Besbas N, Johnson S, Karpman D, Landau D, Licht C, Loirat C, Pecoraro C, Taylor CM, Van de Kar N, Vandewalle J, Zimmerhackl LB (2009) Guideline for the investigation and initial therapy of diarrhea-negative hemolytic uremic syndrome. Pediatr Nephrol 24:687-696

6. Kelly RJ, Hill A, Arnold LM, Brooksbank GL, Richards SJ, Cullen M, Mitchell LD, Cohen DR, Gregory WM, Hillmen P (2011) Long-term treatment with eculizumab in paroxysmal nocturnal hemoglobinuria: Sustained efficacy and improved survival. Blood 117:6786-6792

7. Al-Akash SI, Almond PS, Savell VH Jr, Gharaybeh SI, Hogue C (2011) Eculizumab induces long-term remission in recurrent posttransplant hus associated with $\mathrm{c} 3$ gene mutation. Pediatr Nephrol 26:613-619

8. Gruppo RA, Rother RP (2009) Eculizumab for congenital atypical hemolytic-uremic syndrome. N Engl J Med 360:544-546

9. Mache CJ, Acham-Roschitz B, Fremeaux-Bacchi V, Kirschfink M, Zipfel PF, Roedl S, Vester U, Ring E (2009) Complement inhibitor eculizumab in atypical hemolytic uremic syndrome. Clin J Am Soc Nephrol 4:1312-1316

10. Nester C, Stewart Z, Myers D, Jetton J, Nair R, Reed A, Thomas C, Smith R, Brophy P (2011) Pre-emptive eculizumab and plasmapheresis for renal transplant in atypical hemolytic uremic syndrome. Clin J Am Soc Nephrol 6:1488-1494

11. Nurnberger J, Philipp T, Witzke O, Opazo Saez A, Vester U, Baba HA, Kribben A, Zimmerhackl LB, Janecke AR, Nagel M, Kirschfink M (2009) Eculizumab for atypical hemolytic-uremic syndrome. N Engl J Med 360:542-544

12. Lapeyraque AL, Fremeaux-Bacchi V, Robitaille P (2011) Efficacy of eculizumab in a patient with factor-H-associated atypical hemolytic uremic syndrome. Pediatr Nephrol 26:621-624

13. Weitz M, Amon O, Bassler D, Koenigsrainer A, Nadalin S (2011) Prophylactic eculizumab prior to kidney transplantation for atypical hemolytic uremic syndrome. Pediatr Nephrol 26:13251329

14. Zimmerhackl LB, Hofer J, Cortina G, Mark W, Würzner R, Jungraithmayr TC, Khursigara G, Kliche KO, Radauer W (2010) Prophylactic eculizumab after renal transplantation in atypical hemolytic-uremic syndrome. N Engl J Med 362(18):1746-1748

15. Bouts A, Monnens L, Davin JC, Struijk G, Spanjaard L (2011) Insufficient protection by Neisseria meningitidis vaccination alone during eculizumab therapy. Pediatr Nephrol. doi:10.007/s00477011-1929-3 